\title{
A study of RF power station for microwave rocket launch system
}

\author{
Y.Oda $^{1}$, T. Imai ${ }^{2}$, K. Shimamura ${ }^{2}$, M. Fukunari ${ }^{3}$, H. Katsurayama ${ }^{4}$, N. Ohnishi ${ }^{5}$, and K. Komurasaki ${ }^{6}$ \\ ${ }^{1}$ National Institute for Quantum and Radiological Science and Technology, Naka, Japan, oda.yasuhisa@qst.go.jp \\ ${ }^{2}$ Tsukuba University, Tsukuba, Japan \\ ${ }^{3}$ Research Center for Development of Far-Infrared Region, University of Fukui, Fukui, Japan \\ ${ }^{4}$ Yamaguchi University, Yamaguchi, Japan \\ ${ }^{5}$ Tohoku University, Sendai, Japan \\ ${ }^{6}$ The university of Tokyo, Tokyo, Japan
}

\section{Introduction}

Microwave Rocket is a space launch system which acquires propulsion energy by a high power microwave beam. Since the microwave power station for the launch system, which is the cost driver of the system, will be built on the ground and it will be used for millions of launches, Microwave Rocket is expected to realize a low cost launch system. To deliver microwave power to the vehicle traveling to the satellite orbit, the millimeterwave band high power beam is demanded for long distance beam transmission up to $100 \mathrm{~km}$ and gyrotrons are expected as RF power source. Two kinds of thrust generation mechanism for Microwave Rocket are proposed, namely $\mathrm{CW}$ type and repetitive pulse type. CW type utilizes heat exchange system or steady plasma to heat up propellant by microwave power $[1,2]$. The repetitive pulse type utilizes aerial breakdown which induces shock wave (or blast wave) causes impulsive thrust [3].

There are some studies on Microwave Rocket such as demonstrative launch of a small model [3], theoretical approach of thrust generation model by atmospheric breakdown caused by millimeter wave $[4,5]$, analysis of launch scenario from ground to the orbit [6], and etc. These studies presented the possible requirement of the thrust performance, the momentum coupling coefficient from microwave power to thrust should be 100 $500 \mathrm{~N} / \mathrm{MW}$. When the launch of some hundreds $\mathrm{kg}$ of the vehicle is planned for instance, $100 \mathrm{MW}-1 \mathrm{GW}$ of microwave power in average is required. In this report, a study of GW-class microwave power source design will be presented. We tried to design such a super-high power source by current existing technologies now utilized for the electron cyclotron heating $(\mathrm{ECH})$ system for fusion devices. For example, ECH system for ITER generates $20 \mathrm{MW}$ of $170 \mathrm{GHz}$ RF power by clustering 24 gyrotrons [7]. With consideration of similar technology, the approach of clustering hundreds of MW-class gyrotrons [8$12]$ is evaluated and the necessary technologies for auxiliary system such as microwave transmission and power control are discussed.

\section{Required system for GW-class beam station}

The microwave beam station for Microwave Rocket launching system consists of RF power sources, antenna system, and transmission line as similar to RF heating system.

To generate $100 \mathrm{MW}-1 \mathrm{GW}$ RF power which is requirement for Microwave Rocket launch, the only realistic solution for such high power system is the cluster of MW-class gyrtrons which are available technology today.
Then RF power source of the beam station forms a cluster of some hundreds of gyrotron tubes. The required power level is extremely high in such application, all the gyrotron tubes of the cluster is expected to operate at its maximum operation power to minimize the number of tubes. Then RF power control device, power divider or combiner, is also another key function when the active phased array antenna system requires to control the power of element for instance.

The antenna of the beam station is another key device to realize the requirement of very long distance RF transmission. The beam propagation in free space is usually based on Gaussian beam optics and the diameter of the beam determines its performance. For $100 \mathrm{~km}$ transmission to provide power to the vehicle in launching trajectory, the antenna is expected to have more than $100 \mathrm{~m}$ in diameter. In such huge scaled system, RF transmission technology will dominate the specification of the beam station and it is required to realize higher performance.

\section{RF power source}

The RF power source of the beam station consists of the cluster of gyrotron tubes. This configuration is similar to RF power source of ECH system. One of the restrictions of gyrotron cluster forming is the minimum distance between gyrotrons caused by interaction of magnetic field from neighboring gyrotron system. Kajiwara et al. analyzed the effect of magnetic field of neabering gyrotrons and the magnetic field profile at collector is affected by the neighboring magnet [13]. This may causes the abnormal increase of the collector heat load. The analysis result finally determined the location of gyrotrons in the building. In the GW-class beam station, the minimium distance between gyrotrons is the most dominant parameter to determine the scale of the RF source and the beam station site. The power source builidng can become far larger than the huge antenna system for long distance transmission.

\section{Transmission line}

RF power generated by gryotrons is transmitted to antenna system. Since scale of the antenna system for the beam station is expected greater than $100 \mathrm{~m}$, the distance of RF transmission from output window of gyrotron to antenna element is longer than $100 \mathrm{~m}$ or more. For such long distance transsmission technology is low-loss transmission technology is mandatory.

There is two major method for the long distance lowloss transmission, namely corrugated waveguide and qua- 
si-optical beam transmision. The corrugated waveguide system is widely used in ECH transmission line [14]. In ITER ECH system, its RF transmission disntance and effieicency is more than $160 \mathrm{~m}$ and $89 \%$, respectively [15]. A signel waveguide line can be transmit more than 1 MW of RF power and it is good point to combine the multi beam lines in limited space. On the other hand, it is mandatory to evacuate the waveguide for MW-class transmission and output window is necessary at radiation point. The quasi-optical beam transmission is also used for ECH transmission line such as in W7-X [16]. The beam transmission is able to operate in atmospheric ambient. However the power density is limited and it will be hard to combine many transmission line.

\section{Power control system}

RF power combining and dividing technology is very useful for active control of RF power. The high power RF diplexer which utilizes ring resonation is developed as fast switching device of RF beam for ECH system, such as FADIS by W. Kaparek et al. this technology can be applied for power divider and combiner of beam lines [17]. M. Petelin et al. proposed the cascated resonator which can be utilized for multiplexer [18]. Yet thre requirement of power control is not clearly defined for beam station. Indeed the avilities of these technology is promissing to realize necessary operation of beam power control without changing gyrotron output.

\section{Antenna}

The antenna system for the microwave beam station for rocket launching needs to transmit RF beam for more than $100 \mathrm{~km}$ distance at high power effieciency. For such long distance beam transmission, the scale of antenna system is more than $100 \mathrm{~m}$ in diameter [19]. By using large scle antenna, the power density of the beam near by antenna becomes some-tens $\mathrm{W} / \mathrm{cm}^{2}$ even for the $\mathrm{GW}$ class total output power. This power density level is far lower than MW-class tranmission line. Then generally RF power in the beam line is divided to several elements at antenna.
To launch the rocket, it is necessary to track the beam to launching vehicle which flies away from the station. For this operation, the active phased array technology is expected very usuful because the active phased array antenna can control the beam direction and focusing point by controlling phase and power profile of front plane of the antenna. To realize such operation, power and phase control for high power RF is one of key technologies for develiopment of GW-class beam station system.

\section{References} (2007).

1. A. R. Bruccoleri, et al., J. Propulsion Power, 23, 4, 889

2. G.M. Batanov, et al., Plasma Physics and Plasma Electronics, 241 (1989).

3. T. Nakagawa, et al., J. Spacecraft Rockets, 41, 151, (2004).

4. Y. Oda, et al., J. Propulsion Power, 25, 1, 118 (2009)

5. Y. Oda, et al., J. Appl. Phys., 100, 113308 (2006).

6. H. Katsurayama, et al., J. Space Tech. Sci., 20, 2, 32, (2005).

7. C. Darbos, et al., J. Infrared, Milli. Terahz, Wav.. 37, 1, 4 (2016).

8. K. Sakamoto, et al, Nature Phys., 3, 411 (2007).

9. K. Kajiwara, et al., Fusion Eng. Des., 86, 955 (2011)

10. G.G Denisov, et al., Nuclear Fusion, 48, 054007 (2008).

11. J.Lohr, et al., J. Infrared Milli. Terahz. Wav., 32, 241 (2011).

12. T.Kariya, et al., J. Infrared Milli. Terahz. Wav., 32, 241 (2011).

13. K. Kajiwara et al., Effect of the stray magnetic filed on the gyrotrons for ITER, 34th International Conference on Infrared, Millimeter, and Terahertz Waves (IRMMW 2009), Busan, Korea, 2009. (2008).

14. J. Doane and R. Olstad, Fusion Sci. Tech., 53, 39,

15. F. Gandini et al., Fusion Sci. Tech., 59, 709 (2011).

16. V. Erckmann et al., Fusion Sci. Tech., 52, 291 (2007).

17. W. Kasparek et al, Nuclear Fusion, 48054010 (2008).

18. M. Petelin et al, Plans for quasi-optical experiments at JAEA and NIFS, Proc. 9th International Worksho on Strong Microwaves and Terahertz Waves: Sources and Applications, Nizhny Novgorodo, Russia, 2014.

19. Fukunari et al., J. Infrared, Milli. Terahz, Wav., 38, 2, 176 (2017). 